\title{
Clinical Rheumatology: Quo vadis?
}

\section{Carlos Pineda $^{1}$}

Published online: 2 January 2019

(C) International League of Associations for Rheumatology (ILAR) 2018
Dear Readers,

I am delighted to introduce you to our January 2019 issue of Clinical Rheumatology. This issue represents my first time as the incoming appointed Editor-in-Chief of the journal for the 2019-2023 period.

I would like to acknowledge that the scientific level reached by Clinical Rheumatology during the last 5 years under the leadership of Dr. Luis Espinoza as the past Editorin-Chief is remarkable and lofty in height. I like to continue the laudable work done by my predecessor and colleague. My friendship and closeness to him give me confidence that I will be able to rely on his continuous advice and expert opinion.

I have gathered an elite task force from the previous editorial team with Dr. Adewale Adebajo (UK) as our new Deputy Editor. Drs. Zhanguo Li (China), Cesar Ramos-Remus (Mexico), Tim Jansen (The Netherlands), and Luis J. Jara (Mexico), all of them prominent rheumatologists and well-cited authors, will continue with us as our valuable Associate Editors.

Also, it is my pleasure to introduce Dr. Karina Torralba (USA) as the new Educational Project Coordinator, and Hugo Sandoval, M.Ec., as our local editorial assistant. We will continue to count on the professional support of Ben Bishop, our Publishing Editor (Springer), Wendy Pontefract as Managing Editor, and the rest of the Springer team of associates who will provide continuity and facilitate the proper functioning of the journal.

Also, I will look for an expert in methodology and statistics to fulfill a position in the Editorial Board. This is a priority task that will guarantee the improvement in the quality of research articles published in Clinical Rheumatology in the short, medium, and long term.

Clinical Rheumatology is the official journal of the International League of Associations for Rheumatology

Carlos Pineda

carpineda@yahoo.com

1 Instituto Nacional de Rehabilitación Luis Guillermo Ibarra Ibarra, Calzada México-Xochimilco 289, Colonia Arenal de Guadalupe, CP 143898 Mexico City, Mexico
(ILAR), which is dedicated to publishing original clinical research in the field of rheumatic and musculoskeletal diseases with special emphasis on their clinical aspects. Our journal comes to us from an illustrious predecessor, Acta Rheumatologica Belgica, established in 1945 as the journal of the Belgian Rheumatology Society.

Today, as Dr. Espinoza stated in his incoming Editorial: "Clinical Rheumatology aims to cover and publish all of the recent and established developments in clinical and experimental research, as well as the management and evaluation of diagnostic and treatment procedures linked with the inflammatory, immunologic, metabolic, genetic, and degenerative aspects of rheumatic disorders." [1]

Having been an avid reader, reviewer, board member, and author for Clinical Rheumatology over the years, I appreciate the great honor that accompanies this appointment, since the journal accurately describes and embraces my clinical interests and research predilections for over 30 years as a scholar, clinical researcher, and rheumatologist.

\section{Where do we stand?}

Until some years ago, traditional academic rheumatological research only included articles published in paper journals distributed through subscriptions via postal mail. Today, we live in a connected age. Technological advances allow publications deriving from Rheumatology research to disseminate beyond the physical restrictions imposed by traditional media.

The digitalization of the editorial processes provides great opportunities for those clinical and biomedical areas that depend on audiovisual materials. Clinical Rheumatology has the benefit of the technological advances of electronic files. This feature can add dimension to the author's article, as certain information cannot be printed or is more conveniently presented in electronic form.

Thanks to the changes that have ensued, research currently conducted by rheumatologists incorporates novel issues, among which we highlight: 
1) Big Data analysis and the specialized use of hardware and software in clinical research.

2) New themes dealing with intellectual property, copyrights, patents, and diverse ethical aspects of compliance.

3) Controversial matters related with the interaction of modern Rheumatology with some key stakeholders, e.g., associations of patients, professional societies, governments, universities, and other health professionals and pharmaceutical companies.

4) The impact of the new metrics, such as the H index, the 5year impact factor, Article Influence, the acceptance rate, and the Eigenfactor, and

5) Other initiatives such as cOAlition $\mathrm{S}$ (https://www. scienceeurope.org/coalition-s/) and the San Francisco Declaration on Research Assessment (DORA: https:// sfdora.org/read/), which pursues open access to research publications.

\section{Where we are going?}

Clinical Rheumatology will continue to contribute to the mission of ILAR, in particular to that referred as follows: "Stimulate and support scientific research in musculoskeletal diseases." Inherent our vision, the journal should also seek the following:

1) Improve its current rating among the international publications on Rheumatology; consolidating its global leadership in themes of Clinical Rheumatology, and not leaving to one side other areas of great development and interest for the specialists, such as basic science, and epidemiologic, technological, educational, and rehabilitation issues on rheumatic and musculoskeletal diseases.

2) Increase its impact factor and propose to integrate new metrics.

3) Promote platforms compatible with open access, capable of providing more immediate and public access to a greater number of publications.

4) Encourage the publication of innovative content on-line that eliminates the physical restrictions imposed by the paper format and takes advantage of electronic media.

5) Provide incentives for new mass-diffusion strategies of the contents of Clinical Rheumatology in social networks.

6) Foment a dialog and create permanent scientific exchange networks among its partner organizations (ACR, AFLAR, APLAR, EULAR, and PANLAR), which make up ILAR, to promote its integral development through its body of science and knowledge diffusion: Clinical Rheumatology.

7) Design a support strategy for the publishing of scientific articles and development from young researchers, strengthening their voice, research, and scientific contribution to the journal.

\section{How do we get there from here?}

To comply with this established 5-year vision, I propose to develop the following strategies during the next 5 years:

1. Boost Topical Collections to analyze areas-of-interest and relevance to the specialty as an effective way to disseminate clinical and biomedical advances to our target audience (students, residents, rheumatologists, and allied professionals) produced by the world's leading scientists.

2. Create a new section called Letters of Biomedical and Clinical Research, which informs of the preliminary results of studies, with a maximum of 650 words, 10 references, 2 tables or figures, and a discussion, without a summary or complementary information on-line. The purpose of these Letters will be to present advances in and scoops on highly focused, original clinical research that is easily citable and replicable through other mass media and social networks.

3. Establish a section of Perspectives in Rheumatology, created to share a clinical, methodological, scientific, or ethical point of view regarding provocative or hot topics emerging in the clinical and biomedical practice of Rheumatology, encouraging the participation of the different leagues that make up ILAR, with the purpose of providing each region with a voice in Clinical Rheumatology.

4. Recognize the important work of our select group of reviewers. Through Springer's partnerships with Publons and ORCID, our reviewers will soon be able to receive recognition for their contributions to the Journal. Where reviewers are unable participate in the automated recognition initiatives, we will grant written acknowledgments thanking them for their time, work, and effort devoted the journal.

5. Finally, for Original Research Articles and Brief Reports we will encourage authors to include up to four key points presented in concise sentences, highlighting the contributions of each paper.

These five strategies, in conjunction with our readers, reviewers, Associate Editors and Editorial Board Members, will allow us to continue the Journal's success as one of the leading publications in the field of Rheumatology.

Publisher's note Springer Nature remains neutral with regard to jurisdictional claims in published maps and institutional affiliations.

\section{Reference}

1. Espinoza LR (2014) Clinical Rheumatology: building-up on past success. Clin Rheumatol 33:1. https://doi.org/10.1007/s10067013-2463-4 